\title{
Existence of solutions for fractional $p$-Laplacian problems via Leray-Schauder's nonlinear alternative
}

\author{
Hong Qiu and Mingqi Xiang*
}

\section{"Correspondence:}

mqxiang@cauc.edu.cn

College of Science, Civil Aviation

University of China, Tianjin, 300300,

P.R. China

\section{囪 Springer}

\begin{abstract}
In this paper, we are concerned with the existence of solutions for a class of quasilinear elliptic problems driven by a nonlocal integro-differential operator with homogeneous Dirichlet boundary data. As a particular case, we study the following problem:

$$
(-\Delta)_{p}^{s} u=f(x, u) \text { in } \Omega \text {, }
$$$$
u=0 \quad \text { in } \mathbb{R}^{N} \backslash \Omega \text {, }
$$

where $(-\Delta)_{p}^{s}$ is the fractional $p$-Laplace operator, $\Omega$ is an open bounded subset of $\mathbb{R}^{N}$ with Lipschitz boundary, and $f: \Omega \times \mathbb{R} \rightarrow \mathbb{R}$ is a Carathéodory function. The existence of nonnegative solutions is obtained by using Leray-Schauder's nonlinear alternative.
\end{abstract}

MSC: 35R11; 35A15; 47G20

Keywords: fractional $p$-Laplacian equation; integro-differential operator; Leray-Schauder alternative theorem

\section{Introduction and main results}

Recently, a great deal of attention has been paid to the study of problems involving fractional and nonlocal operators, both in pure mathematical research and in realworld applications, such as optimization, finance, continuum mechanics, phase transition phenomena, population dynamics, minimal surfaces, and game theory, as they are the typical outcome of stochastically stabilization of Lévy processes; see [1-4] and the references therein. Especially, the fractional Laplacian operators of the form $(-\Delta)^{s}$ can be viewed as the infinitesimal generators of stable Lévy processes; see for instance [5]. Some interesting topics concerning the nonlocal fractional operators, such as the nonlinear fractional Schrödinger equation (see [6]), the fractional porous medium equation (see $[7,8]$ ), and so on, have attracted considerable attention. There is no doubt that the literature on fractional and nonlocal operators is quite large; see for example [9-18]. For the basic properties of fractional Sobolev spaces and their applications to elliptic fractional problems, we refer the reader to $[19,20]$ and the references therein.

(c) 2016 Qiu and Xiang. This article is distributed under the terms of the Creative Commons Attribution 4.0 International License (http://creativecommons.org/licenses/by/4.0/), which permits unrestricted use, distribution, and reproduction in any medium, provided you give appropriate credit to the original author(s) and the source, provide a link to the Creative Commons license, and indicate if changes were made. 
In this paper we are interested in the existence of solutions for the following problem:

$$
\left\{\begin{array}{l}
\mathcal{L}_{K} u=f(x, u) \text { in } \Omega, \\
u=0 \quad \text { in } \mathbb{R}^{N} \backslash \Omega,
\end{array}\right.
$$

where $\Omega \subset \mathbb{R}^{N}$ is an open bounded set with Lipschitz boundary $\partial \Omega, f: \Omega \times \mathbb{R} \rightarrow \mathbb{R}$ is a Carathéodory function, and $\mathcal{L}_{K}$ is a nonlocal operator defined as

$$
\mathcal{L}_{K} \varphi(x)=\lim _{\varepsilon \rightarrow 0^{+}} 2 \int_{\mathbb{R}^{N} \backslash B_{\varepsilon}(x)}|\varphi(x)-\varphi(y)|^{p-2}(\varphi(x)-\varphi(y)) K(x-y) d y, \quad x \in \mathbb{R}^{N},
$$

along any $\varphi \in C_{0}^{\infty}\left(\mathbb{R}^{N}\right)$, where $B_{\varepsilon}(x)$ denotes the ball in $\mathbb{R}^{N}$ of radius $\varepsilon>0$ at the center $x \in \mathbb{R}^{N}$ and $K: \mathbb{R}^{N} \backslash\{0\} \rightarrow \mathbb{R}^{+}$is a measurable function with the following property:

$$
\left\{\begin{array}{l}
\gamma K \in L^{1}\left(\mathbb{R}^{N}\right), \text { where } \gamma(x)=\min \left\{|x|^{p}, 1\right\} ; \\
\text { there exist } s \in(0,1) \text { and } K_{0}>0 \\
\quad \text { such that } K(x) \geq K_{0}|x|^{-(N+p s)} \text { for any } x \in \mathbb{R}^{N} \backslash\{0\} .
\end{array}\right.
$$

Throughout the paper, without further mention, we always assume that $0<s<1$ and $1<$ $p<N / s$.

A typical example for $K$ is given by the singular kernel $K(x)=|x|^{-(N+p s)}$. In this case, problem (1.1) becomes

$$
\left\{\begin{array}{l}
(-\Delta)_{p}^{s} u=f(x, u) \quad \text { in } \Omega \\
u=0 \quad \text { in } \mathbb{R}^{N} \backslash \Omega
\end{array}\right.
$$

where $(-\Delta)_{p}^{s}$ is the fractional $p$-Laplace operator; see [19] for more details. When $p=2$, problem (1.3) reduces to the fractional Laplacian problem

$$
\left\{\begin{array}{l}
(-\Delta)^{s} u=f(x, u) \quad \text { in } \Omega \\
u=0 \quad \text { in } \mathbb{R}^{N} \backslash \Omega
\end{array}\right.
$$

A distinguishing characterization of the fractional operator $(-\Delta)^{s}$ in (1.4) is in the nonlocality, in the sense that this operator takes care of the behavior of the solution in the whole space. This is in contrast with the usual elliptic partial differential equations, which are governed by local differential operators like the Laplace operator. Of course, there are other explanations for this feature; see for example [21, 22]. The functional space that takes into account this boundary condition was introduced in [23]. In [22], the authors get the existence of nontrivial weak solutions of problem (1.4) by using the mountain pass theorem. See also [24] for the related discussions. In [25], the author studied the existence of solutions to a variational inequality involving nonlocal elliptic operators and obtained the existence of solutions by using Schauder's fixed point theorem combined with adequate variational arguments and a penalization technique.

In the present paper, motivated by the above papers, we study the existence of weak solutions for problem (1.1) involving nonlocal fractional operator. It is worth noticing that 
there are a few authors addressing the fractional $p$-Laplacian problems by using the nonlinear alternative of Leray-Schauder.

In this spirit, we suppose that $f: \Omega \times \mathbb{R} \rightarrow \mathbb{R}$ is a Carathéodory function satisfying:

$\left(\mathrm{f}_{1}\right)$ there exist $q \in(1, p)$ and $C>0$ such that

$$
|f(x, t)| \leq a(x)+C|t|^{q / q^{\prime}} \quad \text { for all } t \in \mathbb{R} \text { and a.e. } x \in \Omega,
$$

where $q^{\prime}$ satisfies $\frac{1}{q}+\frac{1}{q^{\prime}}=1$, and $a \in L^{q^{\prime}}(\Omega)$, with $a \geq 0$ a.e. in $\Omega$.

A simple example of $f$ is given by $f(x, t)=|t|^{q-2} t+\ln (1+|t|)$ for all $(x, t) \in \Omega \times \mathbb{R}$.

Now we are in a position to give the main result as follows.

Theorem 1.1 Let $K: \mathbb{R}^{N} \backslash\{0\} \rightarrow \mathbb{R}^{+}$be a function satisfying (1.2). Suppose that $f$ satisfies $\left(\mathrm{f}_{1}\right)$. If $1<q<p$, then there exists $R>0$ such that problem (1.1) has a solution $u \in W_{0}$ with $\|u\|_{W_{0}} \leq R$, where the fractional Sobolev space $W_{0}$ will be introduced in Section 2.

This paper is organized as follows. In Section 2, we recall some necessary definitions and properties of the fractional Sobolev space $W_{0}$. In Section 3, applying the properties of the operator $\mathcal{L}_{K}$ and the nonlinear alternative of Leray-Schauder, we obtain the existence of solutions for problem (1.1).

\section{Preliminaries}

In this section, we first recall some basic results, which will be used in the next section. Let $0<s<1<p<\infty$ be real numbers and the fractional critical exponent $p_{s}^{*}$ be defined as

$$
p_{s}^{*}= \begin{cases}\frac{N p}{N-s p} & \text { if } s p<N \\ \infty & \text { if } s p \geq N\end{cases}
$$

In the following, we denote $Q=\mathbb{R}^{2 N} \backslash \mathcal{O}$, where

$$
\mathcal{O}=\mathcal{C}(\Omega) \times \mathcal{C}(\Omega) \subset \mathbb{R}^{2 N}
$$

and $\mathcal{C}(\Omega)=\mathbb{R}^{N} \backslash \Omega$.W is a linear space of Lebesgue measurable functions from $\mathbb{R}^{N}$ to $\mathbb{R}$ such that the restriction to $\Omega$ of any function $u$ in $W$ belongs to $L^{p}(\Omega)$ and

$$
\iint_{Q}|u(x)-u(y)|^{p} K(x-y) d x d y<\infty
$$

The space $W$ is equipped with the norm

$$
\|u\|_{W}=\|u\|_{L^{p}(\Omega)}+\left(\iint_{Q}|u(x)-u(y)|^{p} K(x-y) d x d y\right)^{1 / p} .
$$

It is easy to prove that $\|\cdot\|_{W}$ is a norm on $W$. We shall work in the closed linear subspace

$$
W_{0}=\left\{u \in W: u(x)=0 \text { a.e. in } \mathbb{R}^{N} \backslash \Omega\right\}
$$


endowed with the norm

$$
\|u\|_{W_{0}}:=[u]_{s, p, K}=\left(\iint_{Q}|u(x)-u(y)|^{p} K(x-y) d x d y\right)^{1 / p} .
$$

Then $\left(W_{0},\|\cdot\|_{W_{0}}\right)$ is a uniformly convex Banach space; see [26]. Moreover, $C_{0}^{\infty}(\Omega)$ is dense in $W_{0}$; see [27].

Lemma 2.1 (see Lemma 2.5 of [26]) Let $K: \mathbb{R}^{N} \backslash\{0\} \rightarrow \mathbb{R}^{+}$satisfy (1.2) and let $v_{j}$ be a bounded sequence in $W_{0}$. Then there exists $v \in L^{v}\left(\mathbb{R}^{N}\right)$ with $v=0$ a.e. in $\mathbb{R}^{N} \backslash \Omega$ such that up to a subsequence,

$$
v_{j} \rightarrow v \quad \text { strongly in } L^{v}(\Omega) \text { as } j \rightarrow \infty
$$

for any $v \in\left[1, p_{s}^{*}\right)$.

\section{Proof of the main result}

In this section we prove the existence of solutions to problem (1.1). Our method is based on the properties of the operator $\mathcal{L}_{K}$ and again on the nonlinear alternative of LeraySchauder.

Let $W_{0}^{*}$ denote the dual space of $W_{0}$. We first give some properties to operator $\mathcal{L}_{K}$.

\section{Lemma 3.1}

(1) $\mathcal{L}_{K}: W_{0} \rightarrow W_{0}^{*}$ is a continuous, bounded and strictly monotone operator;

(2) $\mathcal{L}_{K}$ is a mapping of type $\left(S_{+}\right)$, i.e. if $u_{n} \rightarrow u$ in $W_{0}$ and

$$
\limsup _{n \rightarrow \infty}\left\langle\mathcal{L}_{K} u_{n}-\mathcal{L}_{K} u, u_{n}-u\right\rangle \leq 0
$$

then $u_{n} \rightarrow u$ in $W_{0}$

(3) $\mathcal{L}: W_{0} \rightarrow W_{0}^{*}$ is a homomorphism.

Proof (1) Note that, by the Hölder inequality, one has

$$
\begin{aligned}
\left|\left\langle\mathcal{L}_{K} u, v\right\rangle\right| & =\left|\iint_{Q}\right| u(x)-\left.u(y)\right|^{p-2}(u(x)-u(y))(v(x)-v(y)) K(x-y) d x d y \mid \\
& \leq\|u\|_{W_{0}}^{p-1}\|v\|_{W_{0}}
\end{aligned}
$$

for all $u, v \in W_{0}$. Following this inequality, we easily see that $\mathcal{L}_{K}$ is continuous and bounded.

Let us now recall the well-known Simon inequality (see [26] and [28]): for all $\xi, \eta \in \mathbb{R}$, there exists $C_{p}>0$ such that

$$
C_{p}\left(|\xi|^{p-2} \xi-|\eta|^{p-2} \eta\right) \cdot(\xi-\eta) \geq \begin{cases}|\xi-\eta|^{p} & \text { if } p \geq 2 \\ |\xi-\eta|^{2}\left(|\xi|^{p}+|\eta|^{p}\right)^{(p-2) / p} & \text { if } 1<p<2\end{cases}
$$

It follows from (3.1) that the operator $\mathcal{L}_{K}$ is strictly monotone. 
(2) By (1), if $u_{n} \rightarrow u$ and $\lim \sup _{n \rightarrow \infty}\left\langle\mathcal{L}_{K} u_{n}-\mathcal{L}_{K} u, u_{n}-u\right\rangle \leq 0$, then $\lim _{n \rightarrow \infty}\left\langle\mathcal{L}_{K} u_{n}-\right.$ $\left.\mathcal{L}_{K} u, u_{n}-u\right\rangle=0$. Using the well-known vector inequalities (3.1), we obtain, for $p>2$,

$$
\left\|u_{n}-u\right\|_{W_{0}}^{p} \leq C_{p}\left\langle\mathcal{L}_{K} u_{n}-\mathcal{L}_{K} u, u_{n}-u\right\rangle=o(1)
$$

and, for $1<p<2$,

$$
\begin{aligned}
\left\|u_{n}-u\right\|_{W_{0}}^{p} & \leq C_{p}^{p / 2}\left[\left\langle\mathcal{L}_{K} u_{n}-\mathcal{L}_{K} u, u_{n}-u\right\rangle\right]^{p / 2}\left(\left\|u_{n}\right\|_{W_{0}}^{p}+\|u\|_{W_{0}}^{p}\right)^{(2-p) / 2} \\
& \leq C_{p}^{p / 2}\left[\left\langle\mathcal{L}_{K} u_{n}-\mathcal{L}_{K} u, u_{n}-u\right\rangle\right]^{p / 2}\left(\left\|u_{n}\right\|_{W_{0}}^{p(2-p) / 2}+\|u\|_{W_{0}}^{p(2-p) / 2}\right) \\
& \leq C\left[\left\langle\mathcal{L}_{K} u_{n}-\mathcal{L}_{K} u, u_{n}-u\right\rangle\right]^{p / 2}=o(1),
\end{aligned}
$$

where $C>0$ is a constant. Combining (3.2) with (3.3), we obtain $u_{n} \rightarrow u$ in $W_{0}$ as $n \rightarrow \infty$, i.e. $\mathcal{L}_{K}$ is of type $\left(S_{+}\right)$.

(3) By the strictly monotonicity, $\mathcal{L}_{K}$ is an injection. Since

$$
\begin{aligned}
\lim _{\|u\|_{W_{0} \rightarrow \infty}} \frac{\left\langle\mathcal{L}_{K} u, u\right\rangle}{\|u\|_{W_{0}}} & =\lim _{\|u\|_{W_{0} \rightarrow \infty}} \frac{\iint_{Q}|u(x)-u(y)|^{p} K(x-y) d x d y}{\|u\|_{W_{0}}} \\
& =\lim _{\|u\|_{W_{0} \rightarrow \infty}}\|u\|_{W_{0}}^{p-1}=\infty
\end{aligned}
$$

thanks to $1<p<\frac{N}{s}$. Hence $\mathcal{L}_{K}$ is coercive on $W_{0}$. Furthermore, by the Minty-Browder theorem (see Theorem 26A of [29]), we know $\mathcal{L}_{K}$ is a surjection. Thus $\mathcal{L}_{K}$ has an inverse mapping $\mathcal{L}_{K}^{-1}: W_{0}^{*} \rightarrow W_{0}$. Now we check the continuity of $\mathcal{L}_{K}^{-1}$. Assume $g_{n}, g \in W_{0}^{*}$, with $g_{n} \rightarrow g$ in $W_{0}^{*}$. Let $u_{n}=\mathcal{L}_{K}^{-1} g_{n}$ and $u=\mathcal{L}_{K}^{-1} g$, then $\mathcal{L}_{K} u_{n}=g_{n}$ and $\mathcal{L}_{K} u=g$. Clearly, $\left\{u_{n}\right\}$ is bounded in $W_{0}$. Thus there exist $u_{0} \in W_{0}$ and a subsequence of $\left\{u_{n}\right\}$ still denoted by $\left\{u_{n}\right\}$ such that $u_{n} \rightarrow u_{0}$. Since $g_{n} \rightarrow g$, we have

$$
\lim _{n \rightarrow \infty}\left\langle\mathcal{L}_{K} u_{n}-\mathcal{L}_{k} u_{0}, u_{n}-u_{0}\right\rangle=\lim _{n \rightarrow \infty}\left\langle g_{n}, u_{n}-u_{0}\right\rangle=0
$$

In view of $\mathcal{L}_{K}$ is of type $\left(S_{+}\right)$, we get $u_{n} \rightarrow u_{0}$ in $W_{0}$. Moreover, $u=u_{0}$ a.e. in $\Omega$. Hence $u_{n} \rightarrow u$ in $W_{0}$, so that $\mathcal{L}_{K}^{-1}$ is continuous. Thus, we complete the proof.

To prove the existence of solutions for problem (1.1), we need the following theorem.

Theorem 3.1 (Alternative of Leray-Schauder; see [30]) Let $B(0, R)$ denote the closed ball in a Banach space $X,\{u \in X: u \leq R\}$, and let $I: B(0, R) \rightarrow X$ be a compact operator. Then either:

(i) the equation $\lambda I u=u$ has a solution in $B(0, R)$ for $\lambda=1$ or

(ii) there exists $u \in X$ with $\|u\|=R$ satisfying $\lambda \operatorname{Iu}=u$ for some $\lambda \in(0,1)$.

Proof of Theorem 1.1 Following the idea of [31], for simplicity of notation, we set

$$
Y=L^{q}(\Omega), \quad Y^{*}=L^{q^{\prime}}(\Omega), \quad\|\cdot\|_{Y}=\|\cdot\|_{L^{q}(\Omega)} .
$$

By Lemma 2.1 and $1<q<p_{s}^{*}, W_{0}$ is compactly embedded in $Y$. Denote by $i$ the compact injection of $W_{0}$ in $Y$ and by $i^{*}: Y^{*} \rightarrow W_{0}^{*}, i^{*} v=v \circ i$ for all $v \in Y^{*}$, its adjoint. It follows 
from assumption $\left(\mathrm{f}_{1}\right)$ that the Nemytskii operator $N_{f}$ generated by $f,\left(N_{f} u\right)(x)=f(x, u(x))$, is well defined from $Y$ into $Y^{*}$, continuous, and bounded (see for example [32]). In order to prove that problem (1.1) has a weak solution in $W_{0}$ it is sufficient to prove that the equation

$$
\mathcal{L}_{K} u=\left(i^{*} N_{f} i\right) u
$$

has a solution in $W_{0}$. Indeed, if $u \in W_{0}$ satisfies (3.4), then for all $v \in W_{0}$, one has

$$
\left\langle\mathcal{L}_{K} u, v\right\rangle=\left\langle\left(i^{*} N_{f} i\right) u, v\right\rangle=\left\langle N_{f}(i u), i v\right\rangle,
$$

which can be rewritten

$$
\iint_{Q}|u(x)-u(y)|^{p-2}(u(x)-u(y))(v(x)-v(y)) K(x-y) d x d y=\int_{\Omega} f(x, u) v d x,
$$

this means that $u$ is a weak solution in $W_{0}$ to problem (1.1).

By Lemma 3.1, $\mathcal{L}_{K}$ is a homeomorphism of $W_{0}$ onto $W_{0}^{*}$. Equation (3.4) can be equivalently rewritten

$$
u=\mathcal{L}_{K}^{-1}\left(i^{*} N_{f} i\right) u
$$

Therefore, proving problem (1.1) has a weak solution in $W_{0}$ reduces to proving that the compact operator

$$
\mathscr{L}=\mathcal{L}_{K}^{-1}\left(i^{*} N_{f} i\right): W_{0} \rightarrow W_{0}^{*}
$$

has a fixed point.

By Theorem 3.1, a sufficient condition for $\mathscr{L}$ to have a fixed point is that there exists a constant $R>0$ such that

$$
\mathcal{S}=\left\{u \in W_{0}: u=\lambda \mathscr{L} u \text { for some } t \in[0,1]\right\} \subset B(0, R)
$$

Since for $\lambda=0$ the only solution of equation $u=\lambda \mathscr{L} u$ is $u=0$, it is enough to show that there exists a constant such that any $u \in W_{0}$ which satisfies

$$
u=\lambda \mathcal{L}_{K}^{-1}\left[\left(i^{*} N_{f} i\right) u\right]
$$

for some $\lambda \in(0,1]$ belongs to $B(0, R)$.

Indeed, if $u \in W_{0}$ satisfies (3.6) for some $\lambda \in(0,1]$, then we have

$$
\left\langle\mathcal{L}_{K} u, u\right\rangle=\lambda\left\langle\left(i^{*} N_{f} i\right) u, u\right\rangle .
$$

It follows from (3.7) that

$$
\begin{aligned}
\|u\|_{W_{0}}^{p} & =\lambda\left\langle\left(i^{*} N_{f} i\right) u, u\right\rangle \leq\left\langle\left(i^{*} N_{f} i\right) u, u\right\rangle \\
& \leq\left\|i^{*}\right\|\left\|N_{f}(i u)\right\|_{Y^{*}}\|u\|_{W_{0}} .
\end{aligned}
$$


In order to estimate $\left\|N_{f}(i u)\right\|_{Y^{*}}$, we deduce first from assumption $\left(\mathrm{f}_{1}\right)$ that

$$
\left|\left(N_{f} v\right)(x)\right|=|f(x, v)| \leq c_{1}|v(x)|^{q / q^{\prime}}+a(x) .
$$

Hence,

$$
\left\|N_{f} v\right\|_{Y^{*}} \leq\left\|c_{1}|v(x)|^{q / q^{\prime}}+a(x)\right\|_{Y^{*}} \leq c_{1}\|v\|_{Y}^{q-1}+\|a(x)\|_{Y^{*}} .
$$

By taking (3.9) for $v=i u, u \in W_{0}$, we have

$$
\left\|N_{f}(i u)\right\|_{Y^{*}} \leq c_{1}\|i\|^{q-1}\|u\|_{W_{0}}^{q-1}+\|a\|_{Y^{*}}
$$

In particular, if $u \in W_{0}$ and satisfies (3.6) for some $\lambda \in(0,1]$, we derive from (3.8) and (3.10) that

$$
\|u\|_{W_{0}}^{p} \leq\left\|i^{*}\right\|\left(c_{1}\|i\|^{q-1}\|u\|_{W_{0}}^{q-1}+\|a\|_{Y^{*}}\right)\|u\|_{W_{0}}=C_{1}\|u\|_{W_{0}}^{q}+C_{2}\|u\|_{W_{0}},
$$

where $C_{1}=c_{1}\left\|i^{*}\right\|\|i\|^{q}, C_{2}=\left\|i^{*}\right\|\|a\|_{Y^{*}}$. Applying the Young inequality, we get

$$
\|u\|_{W_{0}}^{q} C_{1} \leq \frac{1}{4}\|u\|_{W_{0}}^{p}+4^{\frac{q}{p-q}} C_{1}^{\frac{p}{p-q}}
$$

and

$$
\|u\|_{W_{0}} C_{2} \leq \frac{1}{4}\|u\|_{W_{0}}^{p}+4^{\frac{1}{p-1}} C_{2}^{\frac{p}{p-1}}
$$

Inserting these two inequalities into (3.11), one has

$$
\frac{1}{2}\|u\|_{W_{0}}^{p} \leq 4^{\frac{q}{p-q}} C_{1}^{\frac{p}{p-q}}+4^{\frac{1}{p-1}} C_{2}^{\frac{p}{p-1}}
$$

Set

$$
R=\left(2^{\frac{p+q}{p-q}} C_{1}^{\frac{p}{p-q}}+2^{\frac{p+1}{p-1}} C_{2}^{\frac{p}{p-1}}\right)^{1 / p}
$$

Then $\|u\|_{W_{0}} \leq R$, this implies the set $\mathcal{S}$ is bounded.

\section{Competing interests}

The authors declare that they have no competing interests.

Authors' contributions

All authors contributed equally to the writing of this paper. All authors read and approved the final manuscript.

Acknowledgements

H Qiu was supported by the National Natural Science Foundation of China (No. 11401574 and No. 11326197) and Fundamental Research Funds for the Central Universities (No. 3122013k005). 


\section{References}

1. Caffarelli, L: Nonlocal diffusions, drifts and games. In: Nonlinear Partial Differential Equations. Abel Symposia, vol. 7, pp. 37-52 (2012)

2. Laskin, N: Fractional quantum mechanics and Lévy path integrals. Phys. Lett. A 268, 298-305 (2000)

3. Metzler, R, Klafter, J: The restaurant at the random walk: recent developments in the description of anomalous transport by fractional dynamics. J. Phys. A 37, 161-208 (2004)

4. Caffarelli, L, Roquejoffre, JM, Savin, O: Nonlocal minimal surfaces. Commun. Pure Appl. Math. 63, 1111-1144 (2010)

5. Applebaum, D: Lévy Processes and Stochastic Calculus. Cambridge University Press, Cambridge (2009)

6. Laskin, N: Fractional Schrödinger equation. Phys. Rev. E 66, 056108 (2002)

7. Caffarelli, L, Vázquez, JL: Asymptotic behaviour of a porous medium equation with fractional diffusion. Discrete Contin. Dyn. Syst. 29, 1393-1404 (2011)

8. Vázquez, JL: Nonlinear diffusion with fractional Laplacian operators. In: Nonlinear Partial Differential Equations. Abel Symposia, vol. 7, pp. 271-298 (2012)

9. Molica Bisci, G, Repovs, D: On doubly nonlocal fractional elliptic equations. Atti Accad. Naz. Lincei, Rend. Lincei, Mat. Appl. 26, 161-176 (2015)

10. Molica Bisci, G, Repovs, D: Existence and localization of solutions for nonlocal fractional equations. Asymptot. Anal. 90, 367-378 (2014)

11. Molica Bisci, G: Sequence of weak solutions for fractional equations. Math. Res. Lett. 21, 241-253 (2014)

12. Molica Bisci, G, Repovs, D: Multiple solutions for elliptic equations involving a general operator in divergence form. Ann. Acad. Sci. Fenn., Math. 39(1), 259-273 (2014)

13. Molica Bisci, G, Radulescu, V: Ground state solutions of scalar field fractional Schrödinger equations. Calc. Var. Partial Differ. Equ. 54, 2985-3008 (2015)

14. Ferrara, M, Molica Bisci, G, Repovs, D: Existence results for nonlinear elliptic problems on fractal domains. Adv. Nonlinear Anal. 5(1), 75-84 (2016)

15. Autuori, G, Pucci, P: Elliptic problems involving the fractional Laplacian in $\mathbb{R}^{N}$. J. Differ. Equ. 255, 2340-2362 (2013)

16. Fiscella, A, Valdinoci, E: A critical Kirchhoff type problem involving a nonlocal operator. Nonlinear Anal. 94, 156-170 (2014)

17. Pucci, $P$, Xiang, MQ, Zhang, BL: Multiple solutions for nonhomogeneous Schrödinger-Kirchhoff type equations involving the fractional $p$-Laplacian in $\mathbb{R}^{N}$. Calc. Var. Partial Differ. Equ. 54, 2785-2806 (2015)

18. Xiang, MQ, Zhang, BL, Radulescu, V: Existence of solutions for perturbed fractional $p$-Laplacian equations. J. Differ. Equ. 260, 1392-1413 (2016)

19. Di Nezza, E, Palatucci, G, Valdinoci, E: Hitchhiker's guide to the fractional Sobolev spaces. Bull. Sci. Math. 136, 521-573 (2012)

20. Molica Bisci, G, Radulescu, V, Servadei, R: Variational Methods for Nonlocal Fractional Problems. Encyclopedia of Mathematics and Its Applications, vol. 162. Cambridge University Press, Cambridge (2016). Foreword by J Mawhin. ISBN:9781107111943

21. Iannizzotto, A, Liu, S, Perera, K, Squassina, M: Existence results for fractional $p$-Laplacian problems via Morse theory. Adv. Calc. Var. (2016). doi:10.1515/acv-2014-0024

22. Servadei, R, Valdinoci, E: Mountain pass solutions for non-local elliptic operators. J. Math. Anal. Appl. 389, 887-898 (2012)

23. Servadei, R, Valdinoci, E: Variational methods for non-local operators of elliptic type. Discrete Contin. Dyn. Syst. 33, 2105-2137 (2013)

24. Servadei, R, Valdinoci, E: Léwy-Stampacchia type estimates for variational inequalities driven by (non)local operators. Rev. Mat. Iberoam. 29, 1091-1126 (2013)

25. Xiang, MQ: A variational inequality involving nonlocal elliptic operators. Fixed Point Theory Appl. 2015, 148 (2015). doi:10.1186/s13663-015-0394-2

26. Xiang, MQ, Zhang, BL, Ferrara, M: Existence of solutions for Kirchhoff type problem involving the non-local fractional p-Laplacian. J. Math. Anal. Appl. 424, 1021-1041 (2015)

27. Fiscella, A, Servadei, R, Valdinoci, E: Density properties for fractional Sobolev spaces. Ann. Acad. Sci. Fenn., Math. 40 235-253 (2015)

28. Filippucci, R, Pucci, P, Radulescu, V: Existence and non-existence results for quasilinear elliptic exterior problems with nonlinear boundary conditions. Commun. Partial Differ. Equ. 33, 706-717 (2008)

29. Zeider, E: Nonlinear Functional Analysis and Its Applications. II/B: Nonlinear Monotone Operators. Springer, New York (1990)

30. Dugundji, J, Granas, A: Fixed Point Theory. I. Monografie Matematyczne, vol. 61. PWN, Warsaw (1982)

31. Dinca, G: A fixed point method for the $p(\cdot)$-Laplacian. C. R. Acad. Sci. Paris, Ser. I 347, 757-762 (2009)

32. Fan, XL, Zhang, QH: Existence of solutions for $p(x)$-Laplacian Dirichlet problem. Nonlinear Anal. 52, 1843-1852 (2003) 